\title{
Antiproton radiotherapy: peripheral dose from secondary neutrons
}

\author{
Benjamin P. Fahimian • John J. DeMarco • \\ Roy Keyes • Niels Bassler • Keisuke S. Iwamoto • \\ Maria Zankl • Michael H. Holzscheiter
}

Published online: 29 August 2009

(C) The Author(s) 2009. This article is published with open access at Springerlink.com

\begin{abstract}
The AD-4/ACE collaboration studies the biological effects of antiprotons with respect to a possible use of antiprotons in cancer therapy. In vitro experiments performed by the collaboration have shown an enhanced biological effectiveness for antiprotons relative to protons. One concern is the normal tissue dose resulting from secondary neutrons produced in the annihilation of antiprotons on the nucleons of the target atoms. Here we present the first organ specific Monte Carlo calculations of normal tissue equivalent neutron dose in antiproton therapy through the use of a segmented CT-based human phantom. The MCNPX Monte Carlo code was employed to quantify the peripheral dose for a cylindrical spread out Bragg peak representing a treatment volume of $1 \mathrm{~cm}$ diameter and $1 \mathrm{~cm}$ length in the frontal lobe of a segmented whole-body phantom of a 38 year old male. The secondary neutron organ dose was tallied as a function of energy and organ.
\end{abstract}

Keywords Antiprotons $\cdot$ Particle therapy $\cdot$ Peripheral dose $\cdot$ Neutrons

B. P. Fahimian - J. J. DeMarco · K. S. Iwamoto

Department of Radiation Oncology, University of California, Los Angeles, CA, USA

R. Keyes

Department of Chemical \& Nuclear Engineering, University of New Mexico, Albuquerque, NM, USA

N. Bassler

Deutsches Krebsforschngszentrum Heidelberg (dkfz.), Heidelberg, Germany

M. Zankl

Helmholtz Zentrum Múnchen, Neuherberg, Germany

M. H. Holzscheiter ( $\bowtie)$

Department of Physics \& Astronomy, University of New Mexico, 800 Yale Blvd. NE,

Albuquerque, NM 87131-0001, USA

e-mail: mholzscheiter@phys.unm.edu 


\section{Introduction}

Today, radiation therapy is one of the prominent treatments of cancer, both curative as well as palliative. For conventional photon irradiation, the maximum dose that can be delivered to a tumor is often limited by the tolerance of irradiated adjacent normal tissues. For many types of tumors, this has led to unacceptably low tumor control probability (TCP) and to high levels of morbidity. An alternative approach involves the use of protons and other heavier ions [1-4]. As R. R. Wilson pointed out in 1946 [5], for these charged particles both the amount and rate of energy deposition increase dramatically as the particle nears the end of its range. This results in a large enhancement in absorbed dose at a precise depth in tissue (the Bragg peak) compared with the dose deposited at the entrance to the body. For treatment purposes, the position of the Bragg peak needs to be spread out to cover the tumor volume and the production of such a spread-out Bragg peak (SOBP) results in a build up of plateau dose and hence a reduction in the ratio of dose in the SOBP relative to the plateau. However, in contrast to photons, for a given beam direction, the dose in the SOBP that covers the tumor volume always remains larger than that in the normal tissue entrance region.

High linear energy transfer (LET) particles such as carbon ions produce a much higher ionization density in the Bragg peak region than protons and consequently provide an increase in the relative biological effectiveness (RBE) of the dose deposited in the tumor [6-8]. This provides a potential further therapeutic advantage, especially for tumors that have a large hypoxic fraction or for those that are resistant to conventional radiation [9]. Furthermore, since very little dose is deposited distal to the Bragg peak, charged particles are ideally suited for treatments of tumors close to radiosensitive regions. While these favorable physical and biological characteristics have led to recent developments of proton and heavy ion cancer therapy centers worldwide, the search for possible enhancements of the therapeutic ratio (the ratio of effective dose delivered to the target region to the dose delivered to normal tissue) continues. Antiprotons exhibit similar features as protons while in flight, are intrinsically stable particles, and deposit about twice the energy of protons at the end of range due to annihilation at rest. This additional energy is deposited partially by high LET particles, which increases the biological effective dose even further. The enhanced biological effectiveness of antiproton annihilation in the vicinity of the Bragg peak is believed to be beneficial in the context of increasing tumor control while sparing the surrounding healthy tissue.

The AD-4/ACE collaboration is investigating the potential clinical benefit of antiprotons using the antiproton beam available at the Antiproton Decelerator (AD) at CERN. Initial experiments with $46.7 \mathrm{MeV}$ antiprotons found the biological effective dose ratio (BEDR) between peak and plateau to be 4 times higher for antiprotons than for protons [10]. Recently, we have successfully performed precise measurement of the depth dose profile of antiprotons with ionization chambers [11] and alanine detectors [12], and are therefore now able to extract the relative biological effectiveness (RBE) of antiproton beams from cell survival measurements. RBE values for different cell lines and endpoints can be extracted along the beam path and can be compared with those found for other particle beam modalities such as carbon ions and protons. The results of the physical dose and biological effectiveness measurements are used to benchmark particle transport and radiobiology Monte 
Table 1 Multiplicities for pions, kaons, and heavy ion production in antiproton annihilations in a propane gas target [15]
Bubble chamber simulations 30 in $^{3}$ of liquid Propane, $220 \mathrm{MeV}$ antiproton Beam

\begin{tabular}{lll}
\hline & \multicolumn{2}{l}{ Multiplicities } \\
\cline { 2 - 3 } & Agnew et al. & MCNPX \\
\hline$\pi^{ \pm}$ & $3.38 \pm 0.08$ & $3.09 \pm 0.09$ \\
$\pi^{0}$ & $1.60 \pm 0.50$ & $1.83 \pm 0.05$ \\
$\kappa^{ \pm}$ & $0.08 \pm 0.02$ & $0.11 \pm 0.01$ \\
Heavy ions $,{ }^{3} \mathrm{H},{ }^{3} \mathrm{He}$ & $1.29 \pm 0.07$ & $1.34 \pm 0.07$ \\
\hline
\end{tabular}

Carlo codes. Virtual treatment plans based upon the benchmarked Monte Carlo codes can then help to identify those tumor incidents where antiproton therapy may offer a decisive advantage over other modalities [13].

One important issue in the assessment of a new treatment modality is any background dose deposited outside of the primary target. In antiproton annihilation this background results from medium and long-range annihilation products, predominantly charged pions, high energy gammas, and neutrons. We have launched a major effort to address this effect using experiments and Monte Carlo calculations utilizing a variety of code packages. In this report we concentrate on the example of neutron equivalent dose to out-of-field organs using MCNPX v26F [14].

\section{Calculation of neutron equivalent dose}

When an antiproton annihilates after being captured by the nucleus of a target atom, a number of neutrons, depending on the target nucleus, are emitted. The energy spectrum of these neutrons spans from thermal energies up to several hundred $\mathrm{MeV}$, with a mean energy of approximately $50 \mathrm{MeV}$. The biological efficiency of these neutrons varies with energy and the International Commission on Radiological Protection (ICRP) has published weighting factors vs. energy for neutrons in human tissue. Because of the high biological effectiveness of neutrons and their abundance in the particle spectrum we decided to study the effect in detail.

\subsection{Benchmarking of MCNPX}

MCNPX is a Monte Carlo transport package that is widely used in clinical applications. Nevertheless, when applying it to antiproton therapy the code must be benchmarked against available experimental data to assure that correct physical models and transport parameters are utilized and the description of the annihilation process is complete. We have simulated both the bubble chamber experiments by Agnew et al. [15] and the neutron multiplicity for annihilation of antiprotons at rest in the center of slabs of ${ }^{63} \mathrm{Cu}$ reported by Polster et al. [16]. The physical models used in the simulations consisted of the Bertini model for nucleons and pions, the LAQGSM model was used for all heavy ions and light ions above $940 \mathrm{MeV} /$ nucleon, and the ISABEL model was used for the remaining the light ions [15]. Our calculations for Agnews work agreed well with the experimental results after appropriate changes to the set cut-off energies were incorporated in the code [Table 1]. 

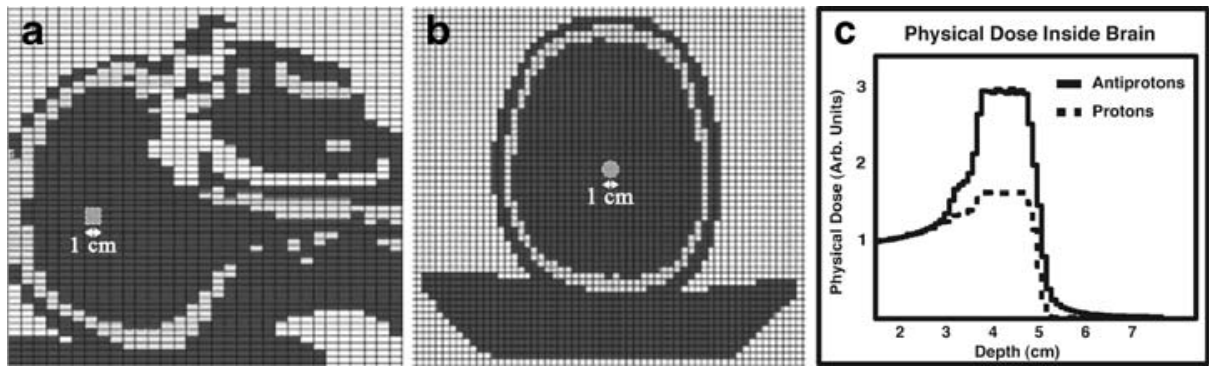

Fig. 1 a, b Sagittal and transverse views of the voxelized phantom's head and the PTV c Resulting depth dose profiles (physical dose only) in the beam direction inside the brain for the antiproton and proton simulations, normalized to the same entrance dose

We also found good agreement between the general shape of the energy spectrum of neutrons produced in the annihilation of antiprotons at rest in the center of a ${ }^{63} \mathrm{Cu}$ slab obtained by MCNPX and the analytical function given by Polster et al., but a slight overshoot in two distinct energy regions resulted in a higher overall neutron multiplicity than reported by Polster [16]. The overshoot may be related to the difficulties and unknowns in modeling the Polster experiments; however, the results were found to be adequate for the purposes of establishing an upper estimate of the neutron equivalent dose.

\subsection{Virtual antiproton irradiation of a voxelized phantom}

To model the biological effect of a patient treatment with antiprotons we used a voxelized phantom of a 38 year old living man (Golem) of approximately the same dimensions as the ICRP Man [17]. Golem has a weight of $68.9 \mathrm{~kg}$ and a height of $176 \mathrm{~cm}$. The voxel size is $34.6 \mathrm{~mm}^{3}$. The planning target volume (PTV) consisted of a cylinder of $1 \mathrm{~cm}$ diameter and $1 \mathrm{~cm}$ length situated in the central lobe as shown in Fig. 1. Comparative simulations were performed for both antiprotons and protons using active beam modulation. Spread-out Bragg peaks were generated by modulating the intensities and beam energies between 74 and $86 \mathrm{MeV}$, resulting in the profiles shown in Fig. 1c.

The dose deposited by neutrons resulting from the annihilation in the target was tallied for the individual organs of Golem as a function of neutron energy. The value for the normal tissue dose received by the brain was obtained by subtracting the tumor dose estimated using a mesh tally covering the target volume with a $1.5 \mathrm{~mm}$ margin from the total dose. Using the neutron dose as a function of energy obtained from MCNPX and the neutron radiation weighting factors given as a function of energy by ICRP, the physical dose in the different organs was then transformed to equivalent dose by integrating the product of the physical dose and radiation weighting factors over all energy bins. The resulting organ equivalent doses per gray of physical dose delivered to the target region for the antiproton and proton treatments are presented in Table 2 for a number of organs (simulation error implied by decimal point). 
Table 2 Comparison of neutron equivalent dose for selected organs, normalized to unit physical dose to the tumor for the given antiproton and proton treatments

\begin{tabular}{lll}
\hline Comparison of neutron equivalent dose per Gy in SOBP (Sv/Gy) & \\
\hline & Antiprotons (ICRP 92) & Protons (ICRP 92) \\
\hline Brain & $8.2 \times 10^{-4}$ & $1.2 \times 10^{-5}$ \\
Thyroid & $2.8 \times 10^{-5}$ & $3.1 \times 10^{-7}$ \\
Bone & $2.2 \times 10^{-5}$ & $3.8 \times 10^{-7}$ \\
Lung & $9.7 \times 10^{-6}$ & $8.4 \times 10^{-8}$ \\
Liver & $4.8 \times 10^{-6}$ & $3.8 \times 10^{-8}$ \\
Ceacum, colon, rectum & $2.4 \times 10^{-6}$ & $1.4 \times 10^{-8}$ \\
\hline
\end{tabular}

For the purpose of generality, the enhanced SOBP RBE of antiprotons is not incorporated in this table

\section{Conclusions}

Using an anthropomorphic phantom, the first Monte Carlo estimates of tissue specific neutron equivalent dose in antiproton therapy have been produced. For scanning energy modulation, and a $1 \mathrm{~cm}$ cylindrical PTV in the brain, the calculated neutron equivalent doses for the organs of Golem range from .0001 to $.8 \mathrm{mSv}$ per Gy of SOBP, depending on the proximity to the SOBP. In order to properly compare these results with that of proton therapy, the enhanced RBE for antiprotons in the SOBP must be considered in addition to the physical dose normalized comparisons of Table 2. Using the results from [10] this suggests that the neutron equivalent dose for antiproton therapy is roughly 60 times higher than what is obtained with protons for the given treatment plan. Here it should be noted that most proton therapy centers currently use passive scattering methods for beam delivery which increases the radiation level to the patient by 1-2 orders of magnitude [18]. Such delivery techniques are precluded for antiprotons by both the scarcity of these exotic particles and the high production rate for neutrons and pions by antiprotons interacting with the material of apertures and compensators. Future work will include a treatment plan which takes into account the RBE variation along the beam path. This will lower the actual number of antiprotons needed to deliver the identical biological effective dose to the tumor as for the proton treatment prescribed and will therefore reduce the number of neutrons produced.

In addition to the peripheral neutron dose described here there is also a background of pions and high-energy photons produced in the antiproton annihilation event. Preliminary calculations show that the physical dose for these components is similar to the neutron dose, but as these particles have a low linear energy transfer we expect the equivalent dose to be below the neutron dose. Monte Carlo studies of these issues are ongoing in parallel to experimental studies of biological effects in the peripheral region.

Acknowledgements This work has been performed in the context of the AD-4/ACE collaboration ${ }^{1}$ and the authors are grateful for the many fruitful discussions with other collaboration members. NB thanks the Danish Cancer Society for supporting this project with a grant.

$\overline{{ }^{1} \text { http://www.phys.au.dk/ hk/ad} 4 h o m e p a g e . h t m l ~}$ 
Open Access This article is distributed under the terms of the Creative Commons Attribution Noncommercial License which permits any noncommercial use, distribution, and reproduction in any medium, provided the original author(s) and source are credited.

\section{References}

1. Levin, W.P., Kooy, H., Loeffler, J.S., DeLaney, T.F.: Proton beam therapy. Br. J. Cancer 93, 849-854 (2005)

2. Suit, H., Goldberg, S., Niemierko, A., Trofimov, A., Adams, J., Paganetti, H., Chen, G.T., Bortfeld, T., Rosenthal, S., Loeffler, J., Delaney, T.: Proton beams to replace photon beams in radical dose treatments. Acta Oncol. 42, 800-808 (2003)

3. Schulz-Ertner, D., Nikoghosyan, A., Thilmann, C., Haberer, T., Jäkel, O., Karger, C., Scholz, M., Kraft, G., Wannenmacher, M., Debus, J.: Carbon ion radiotherapy for chordomas and low-grade chondrosarcomas of the skull base. Results in 67 patients. Strahlenther. Onkol. 179, 598-605 (2003)

4. Mazeron, J.J., Noel, G., Feuvret, L., Calugaru, V., Racadot, S.: Clinical complementarities between proton and carbon therapies. Radiother. Oncol. 73, S50-S52 (2004)

5. Wilson, R.R.: Radiological use of fast protons. Radiology 47, 487-491 (1946)

6. Blakely, E.A., Tobias, C.A., Yang, T.C., Smith, K.C., Lyman, J.T.: Inactivation of human kidney cells by high-energy monoenergetic heavy-ion beams. Radiat. Res. 80, 122-160 (1979)

7. Weyrather, W.K., Kraft, G.: RBE of carbon ions: experimental data and the strategy of RBE calculation for treatment planning. Radiother. Oncol. 73, S161-S169 (2004)

8. Weyrather, W.K., Ritter, S., Scholz, M., Kraft, G.: RBE for carbon track-segment irradiation in cell lines of differing repair capacity. Int. J. Radiat. Biol. 75, 1357-1364 (1999)

9. Svensson, H., Ringborg, U., Naslund, I., Brahme, A.: Development of light ion therapy at the Karolinska Hospital and Institute. Radiother. Oncol. 73, S206-S210 (2004)

10. Holzscheiter, M.H., Bassler, N., Agazaryan, N., Beyer, G., Blackmore, E., DeMarco, J.J., Doser, M., Durand, R.E., Hartley, O., Iwamoto, K.S., Knudsen, H.V., Landua, R., Maggiore, C.J., McBride, W.H, Møller, S.P., Petersen, J., Skarsgard, L.D., Smathers, J.B., Solberg, T.D., Uggerhøj, U.I., Vranjes, S., Withers, H.R., Wong, M., Wouters, B.G.: The biological effectiveness of antiproton irradiation. Radiother. Oncol. 81, 233-242 (2006)

11. Bassler, N., Holzscheiter, M.H., Jäkel, O., Knudsen, H.V., Kovacevic, S., the AD-4/ACE Collaboration: The antiproton depthdose curve in water. Phys. Med. Biol. 53, 793-805 (2008)

12. Bassler N., Hansen J.W., Palmans H., Holzscheiter M.H., Kovacevic S., the AD-4/ACE Collaboration: The antiproton depthdose curve measured with alanine detectors. Nucl Instrum Methods Phys B 266, 929-936 (2008)

13. Bassler, N., Alsner, J., Beyer, G., DeMarco, J.J., Doser, M., Hajdukovic, D., Hartley, O., Iwamoto, K.S., Jäkel, O., Knudsen, H.V., Kovacevic, S., Møller, S.P., Overgaard, J., Petersen, J.B., Solberg, T.D., Sørensen, B.S., Vranjes, S., Wouters, B.G., Holzscheiter, M.H.: Antiproton radiotherapy. Radiother. Oncol. 86, 1419 (2008)

14. Waters, L.S.: MCNPX Users Manual, Version 2.3.0. Los Alamos Unclassified Research Document LA-UR-02-2607 (2002)

15. Agnew, L.E., Elioff Jr., T., Fowler, W.B., Lander, R.L., Powell, W.M., Segré, E., Steiner, H.M., White, H.S., Wiegand, C., Ypsilantis, T.: Antiproton interactions in hydrogen and carbon below 200 MeV. Phys. Rev. 118, 1371-1391 (1960)

16. Polster, D., Hilscher, D., Rossner, H., Schmid, W., Baumann, P., Daniel, H., von Egidy, T., Hartmann, F.J., Hofmann, P., Kim, Y.S., Lotfranei, M.S.: Spectra and multiplicities of n, p, d, $\mathrm{t}, \mathrm{K}^{ \pm}, \pi^{ \pm}$from antiproton annihilation in $\mathrm{Cu}$ and U. Phys. Lett., B 300, 317-321 (1993)

17. Petoussi-Henss, N., Zankl, M., Fill, U., Regulla, D.: The GSF family of voxel phantoms. Phys. Med. Biol. 47, 89-106 (2002)

18. Xu, X.G., Bednarz, B., Paganetti, H.: Phys. Med. Biol. 53, R 193-R 241 (2008) 